DTP $/ 99 / 112$

hep-ph/9912300

December 1999

\title{
Sudakov logarithm resummation for vector boson production at hadron colliders
}

\author{
A Kulesza $†$ and W J Stirling $† \dagger$ \\ $\dagger$ Department of Physics, University of Durham, Durham DH1 3LE, U.K. \\ $\ddagger$ Department of Mathematical Sciences, University of Durham, Durham \\ DH1 3LE, U.K.
}

\begin{abstract}
A complete description of $W$ and $Z$ boson production at highenergy colliders requires the resummation of large Sudakov logarithms which dominate the production at small transverse momentum. Currently there are two techniques for performing this resummation: impact parameter space and transverse momentum space. We argue that the latter can be formulated in a way which retains the advantages of the former, while at the same time allowing a smooth transition to finite order dominance at high transverse momentum.
\end{abstract}

\section{Introduction}

The search for a correct description of initial gluon radiation has recently become a very active field of theoretical research, stimulated by the large amount of experimental data now available. In particular, gluon radiation plays an important role in vector boson $(W, Z)$ production at hadron colliders, i.e. processes that provide precision measurements of Standard Model parameters and appear to be significant backgrounds to new physics phenomena. For example, one of the methods of determining $M_{W}$ requires a very accurate description of the $W$ 's transverse momentum distribution. At small transverse momentum $\left(q_{T}\right)$, this distribution is dominated by large logarithms $\ln \left(Q^{2} / q_{T}^{2}\right)$ which are directly related to the initial emission of soft gluons. Therefore at sufficiently small $q_{T}$ fixed-order perturbation theory breaks down and the logarithms must be resummed.

The origin of the large logarithms is visible already at the leading-order (LO) level - the contribution from real emission diagrams for $q \bar{q} \rightarrow V g$ contains a term of the form $\frac{\alpha_{S} C_{F}}{\pi q_{T}^{2}} \ln \left(\frac{Q^{2}}{q_{T}^{2}}\right)$.

In the case of higher-order contributions, where more gluons are emitted, the logarithmic divergence becomes even stronger. It can be shown that in the approximation of soft and collinear gluons with strongly ordered transverse momenta $k_{T}$, i.e.

$$
k_{T_{i 1}}^{2} \ll k_{T_{i 2}}^{2} \ll \ldots \ll k_{T_{i N}}^{2} \lesssim q_{T}^{2} \ll Q^{2}
$$

the dominant contributions to the cross section are

$$
\begin{aligned}
\frac{1}{\sigma_{0}} \frac{d \sigma}{d q_{T}^{2}}= & \frac{1}{q_{T}^{2}}\left[\alpha_{s} \frac{A^{(1)}}{2 \pi} \ln \left(\frac{Q^{2}}{q_{T}^{2}}\right)-\alpha_{s}^{2} \frac{\left(A^{(1)}\right)^{2}}{8 \pi^{2}} \ln ^{3}\left(\frac{Q^{2}}{q_{T}^{2}}\right)+\ldots\right. \\
& \left.+\alpha_{s}^{N} \frac{(-1)^{N-1}\left(A^{(1)}\right)^{N}}{2^{2 N-1}(N-1) ! \pi^{N}} \ln ^{2 N-1}\left(\frac{Q^{2}}{q_{T}^{2}}\right)+\ldots\right],
\end{aligned}
$$

$\S$ Contribution to the Proceedings of the UK Phenomenology Workshop on Collider Physics, Durham, UK, 19-24 September 1999, to be published in J. Phys. G. 
where $A^{(1)}=2 C_{F}$. Due to the $\alpha_{s}^{N} \ln ^{2 N-1}\left(Q^{2} / q_{T}^{2}\right)$ structure, this approximation is commonly known as the Double Leading Logarithm Approximation (DLLA). Although the approximation of truncating the series in (2) at finite order clearly breaks down once $\alpha_{s} \ln ^{2}\left(Q^{2} / q_{T}^{2}\right) \sim 1$, the form of the coefficients allows (2) to be resummed, giving a so-called Sudakov factor [1] :

$$
\frac{1}{\sigma_{0}} \frac{d \sigma}{d q_{T}^{2}}=\frac{\alpha_{s} A}{2 \pi q_{T}^{2}} \ln \left(\frac{Q^{2}}{q_{T}^{2}}\right) \exp \left(\frac{-\alpha_{s} A}{4 \pi} \ln ^{2}\left(\frac{Q^{2}}{q_{T}^{2}}\right)\right) .
$$

Resummation of (2) to all orders gives a finite but unphysically suppressed result in the small $q_{T}$ limit. This suppression is caused by the vanishing of strongly-ordered phase-space, in which overall transverse momentum conservation is ignored. The result in (3) corresponds to a configuration in which a single soft gluon balances the $W$ transverse momentum, giving the overall $\ln \left(Q^{2} / q_{T}^{2}\right) / q_{T}^{2}$ term, while all other gluons have transverse momenta $\ll q_{T}$. This is not the dominant configuration in the small $q_{T}$ limit. Equally important are non-strongly-ordered contributions corresponding to the emission of soft $\left(\sim q_{T}\right)$ gluons whose transverse momenta add vectorially to give the overall $q_{T}$ of the $W$. Although such contributions are formally subleading order-by-order, they do dominate the cross section in the region where the Sudakov form factor suppresses the (formally) leading DLLA contributions. The non-leading 'kinematical' logarithms are correctly taken into account by imposing transverse momentum conservation (rather than strong ordering), and this is most easily achieved in Fourier transform space ( $b$-space).

\section{2. $b$-space method}

The $b$-space method is based on introducing a 2-dimensional impact parameter vector $\boldsymbol{b}$, the Fourier conjugate of $\boldsymbol{q}_{T}$ [2]. First, the cross-section (with a delta function for transverse momentum conservation included) is Fourier transformed, then the conjugated cross section $\hat{\sigma}(b)$ is computed, and finally this is transformed back to momentum space. The advantage of the $b$-space method is that the soft gluon factorisation property is retained even in the presence of transverse momentum conservation:

$$
\delta^{(2)}\left(\sum_{i=1}^{N} \boldsymbol{k}_{\boldsymbol{T}_{\boldsymbol{i}}}-\boldsymbol{q}_{\boldsymbol{T}}\right)=\int d^{2} b \frac{1}{4 \pi^{2}} e^{-i \boldsymbol{b} \boldsymbol{q}_{\boldsymbol{T}}} \prod_{i=1}^{N} e^{i \boldsymbol{b} \boldsymbol{k}_{\boldsymbol{T}_{\boldsymbol{i}}}} .
$$

This allows for the development of a general expression resumming all terms of the perturbation series which are at least as singular as $1 / q_{T}^{2}$ when $q_{T} \rightarrow 0$ [3] (at the parton level):

$$
\frac{d \sigma}{d q_{T}^{2}}=\frac{\sigma_{0}}{2} \int_{0}^{\infty} b d b J_{0}\left(q_{T} b\right) e^{S\left(b, Q^{2}\right)},
$$

with $\sigma_{0}=4 \pi \alpha^{2} /(9 s)$, and where

$$
\begin{aligned}
& S\left(b, Q^{2}\right)=-\int_{\frac{b_{0}^{2}}{b^{2}}}^{Q^{2}} \frac{d \bar{\mu}^{2}}{\bar{\mu}^{2}}\left[\ln \left(\frac{Q^{2}}{\bar{\mu}^{2}}\right) A\left(\alpha_{S}\left(\bar{\mu}^{2}\right)\right)+B\left(\alpha_{S}\left(\bar{\mu}^{2}\right)\right)\right], \\
& A\left(\alpha_{S}\right)=\sum_{i=1}^{\infty}\left(\frac{\alpha_{S}}{2 \pi}\right)^{i} A^{(i)}, \quad B\left(\alpha_{S}\right)=\sum_{i=1}^{\infty}\left(\frac{\alpha_{S}}{2 \pi}\right)^{i} B^{(i)},
\end{aligned}
$$

with $b_{0}=2 \exp \left(-\gamma_{E}\right)$. The first two coefficients in each series (7) can be obtained [4] from the exact fixed-order perturbative calculation in the high $q_{T}$ region by comparing 
the logarithmic terms therein with the corresponding logarithms generated by the first three terms of the expansion of $\exp \left(S\left(b, Q^{2}\right)\right)$ in (5).

Although the $b$-space method succeeds in recovering a finite, positive result in the $q_{T} \rightarrow 0$ limit, it suffers from several drawbacks. The first is the difficulty of matching the resummed and fixed-order predictions. Since the resummation is performed in the Fourier-conjugated space one loses control over which logarithmic terms (in $q_{T}$-space) are taken into account. Therefore there is no unambiguous prescription for matching; existing prescriptions require 'unsmooth' switching from resummed to fixed-order calculation at some value of $q_{T}$. Secondly, since the integration in (5) extends from 0 to $\infty$, it is impossible to make predictions for any $q_{T}$ without having a prescription for how to deal with the non-perturbative regime of large $b$. One prescription is to artificially prevent $b$ from reaching large values by replacing it with a new variable $b_{*}$ and by parametrising the non-perturbative large- $b$ region in terms of a form factor. The cross section then reads

$$
\begin{aligned}
\frac{d \sigma}{d q_{T}^{2}} \sim \sum_{q} & e_{q}^{2} \int_{0}^{1} d x_{A} d x_{B} \delta\left(x_{A} x_{B}-\frac{Q^{2}}{S}\right) \frac{1}{2} \int_{0}^{\infty} d b b J_{0}\left(q_{T} b\right) \\
& \times\left\{f_{q / A}\left(x_{A}, \frac{b_{0}}{b_{*}}\right) f_{\bar{q} / B}\left(x_{B}, \frac{b_{0}}{b_{*}}\right) F_{a b}^{N P}\left(Q, b, x_{A}, x_{B}\right) \exp \left[\mathcal{S}\left(b_{*}, Q\right)\right]+(q \leftrightarrow \bar{q})\right\} .
\end{aligned}
$$

where, for example, the 'freezing' of $b$ at $b_{*}$ is achieved by

$$
b_{*}=\frac{b}{\sqrt{1+\left(b / b_{\lim }\right)^{2}}}, \quad \quad b_{*}<b_{\lim },
$$

with the parameter $b_{\lim } \sim 1 / \Lambda_{\mathrm{QCD}}$ separating perturbative and non-perturbative physics. The detailed form of the non-perturbative function $F_{a b}^{N P}$ remains a matter of theoretical dispute (for a review see [5]). In a very simple model in which the nonperturbative contribution arises from a Gaussian 'intrinsic' $k_{T}$ distribution, one would have $F \sim \exp \left(-\kappa b^{2}\right)$. The data are not inconsistent with such a form, but suggest that the parameter $\kappa$ may have some dependence on $Q$ and $x$.

\section{3. $q_{T}$-space method}

The difficulties mentioned above could in principle be overcome if one had a method of performing the calculations directly in transverse momentum space. Given an insight into which logarithmic terms are resummed, it should be fairly straightforward to perform matching with the fixed-order result. Moreover, the non-perturbative input would be required in (and would affect) only the small $q_{T}$ region.

Three techniques have been proposed for carrying out resummation in $q_{T}$-space [6, 8, 8]. For a detailed discussion of the differences between them the reader is referred to [9]. The starting point for all techniques is the general expression in impact parameter (b) space for the vector boson transverse momentum distribution in the Drell-Yan process [3] at the quark level. In the approach of [8], the final result (in the simplest case with fixed coupling $\alpha_{S}$ and retaining only the leading coefficient $A^{(1)}$ in the $A$ and $B$ series of (更) is of the form

$$
\frac{1}{\sigma_{0}} \frac{d \sigma}{d q_{T}^{2}}=\frac{\lambda}{q_{T}^{2}} e^{\frac{-\lambda}{2} L^{2}} \sum_{N=1}^{\infty} \frac{(-2 \lambda)^{(N-1)}}{(N-1) !} \sum_{m=0}^{N-1}\left(\begin{array}{c}
N-1 \\
m
\end{array}\right) L^{N-1-m}\left[2 \tau_{N+m}+L \tau_{N+m-1}\right] .
$$


Here $L=\ln \left(Q^{2} / q_{T}^{2}\right), \lambda=\alpha_{S} C_{F} / \pi$, and the numbers $\tau_{m}$ are defined by 凹

$$
\tau_{m} \equiv \int_{0}^{\infty} d y J_{1}(y) \ln ^{m}\left(\frac{y}{b_{0}}\right) .
$$

The $\tau_{m}$ can be calculated explicitly using the generating function

$$
\sum_{m=0}^{\infty} \frac{1}{m !} t^{m} \tau_{m}=e^{t \gamma_{E}} \frac{\Gamma\left(1+\frac{t}{2}\right)}{\Gamma\left(1-\frac{t}{2}\right)}=\exp \left[-2 \sum_{k=1}^{\infty} \frac{\zeta(2 k+1)}{2 k+1}\left(\frac{t}{2}\right)^{2 k+1}\right],
$$

so that e.g. $\tau_{0}=1, \tau_{1}=\tau_{2}=0, \tau_{3}=-\frac{1}{2} \zeta(3)$ etc.

Notice that by setting all $\tau_{m}$ coefficients (except $\tau_{0}$ ) to zero one would immediately recover the DLLA form (8). Since there are no explicit subleading logarithms in (5), other than those related to kinematics, the presence of the $\tau_{m}$ coefficients must correspond to relaxing the strong-ordering condition. This can be checked explicitly by performing the 'exact' $\mathcal{O}\left(\alpha_{s}^{2}\right)$ calculation in transverse momentum space:

$$
\begin{aligned}
\int d^{2} k_{T 1} d^{2} k_{T 2} & {\left[\frac{\ln \left(Q^{2} / k_{T 1}^{2}\right)}{k_{T 1}^{2}}\right]_{+}\left[\frac{\ln \left(Q^{2} / k_{T 2}^{2}\right)}{k_{T 2}^{2}}\right]_{+} \delta^{(2)}\left(\boldsymbol{k}_{\boldsymbol{T} \mathbf{1}}+\boldsymbol{k}_{\boldsymbol{T} \mathbf{2}}-\boldsymbol{q}_{\boldsymbol{T}}\right) } \\
= & \frac{\pi}{q_{T}^{2}}\left(-L^{3}+4 \zeta(3)\right) .
\end{aligned}
$$

Strong ordering is equivalent to replacing the $\delta$-function by $\delta^{(2)}\left(\boldsymbol{k}_{\boldsymbol{T} \mathbf{1}}-\boldsymbol{q}_{\boldsymbol{T}}\right) \times$ $\theta\left(k_{T 1}^{2}-k_{T 2}^{2}\right)+(1 \leftrightarrow 2)$. This gives only the leading $L^{3}$ term on the right-hand side. The $\zeta(3)$ term represents the first appearance of the (kinematic) $\tau_{3}$ coefficient of (8).

In principle the formalism presented above allows for an inclusion of any number of such subleading kinematic logarithms, defined by the cut-off value $N_{\max }$. Figure 1 shows that for small values of $q_{T}$ the approximation of the $b$-space result improves with increasing $N_{\max }$. Therefore by retaining sufficiently many terms one can obtain a good approximation (i.e. adequate for phenomenological purposes) to the $b$-space result by summing logarithms directly in $q_{T}$ space. The technique developed so far

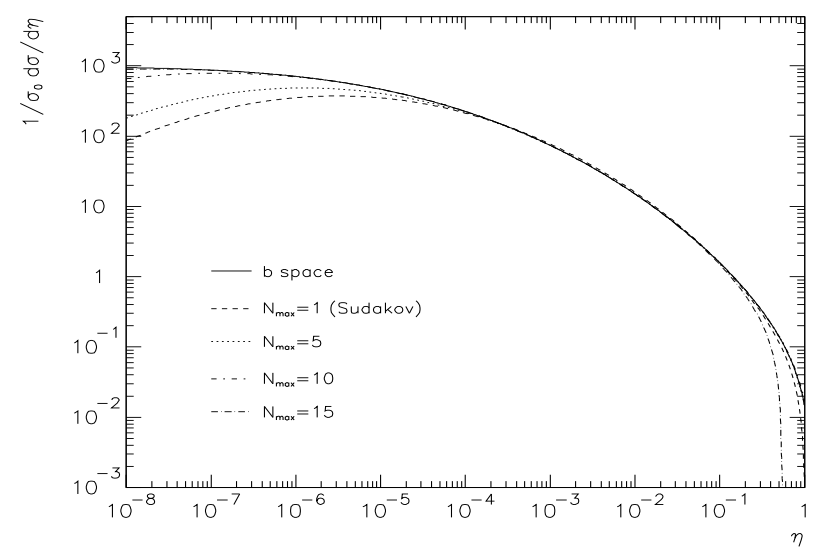

Figure 1. The $b$-space result (parton level, fixed coupling, only $A^{(1)}$ ) compared to the expression (B), calculated for various values of $N_{\max }$. Here $\eta=q_{T}^{2} / Q^{2}$.

$\|$ The $\tau_{m}$ are called $\bar{b}_{m}(\infty)$ in 8 .

I However, due to the lack of knowledge of $A^{(3)}, B^{(3)}$, etc. it is only possible to obtain a result where not more than the first four 'towers' of logarithms are fully resummed, see [8]. 
can be extended to include subleading $A$ and $B$ coefficients, the running coupling and parton distributions, thus yielding a 'realistic' expression for the hadron-level cross section. The result is too lengthy to reproduce here, but can be found in [8, 10]. A comparison between the (Tevatron) D0 data 11] on the $Z$ transverse momentum distribution and two of the $q_{T}$-space predictions $] 6$, 8] is shown in Figure 2. No nonperturbative contribution is included, and in fact the fits can be improved slightly by including a modest amount of Gaussian intrinsic $k_{T}$ smearing, as discussed above.

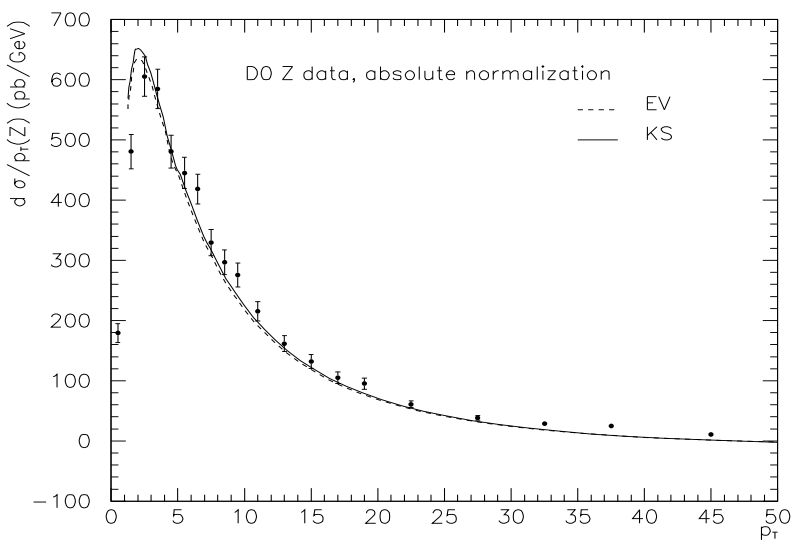

Figure 2. Comparison of the theoretical predictions derived in the Ellis-Veseli (EV) [6] and Kulesza-Stirling (KS) \&8, 10] approach with D0 data 11.

\section{Conclusions}

We have discussed two methods of performing resummation in vector boson production at hadron colliders. We have argued in favour of the $q_{T}$-space method, which can be extended to systematically take into account subleading logarithms of kinematic origin. However more work is required. Although the $q_{T}$-space method provides a simple matching prescription, the form of the non-perturbative function in this approach remains an open theoretical issue. In particular, the current lack of understanding of the $x$ and $Q$ dependence of the non-perturbative contribution is a serious limiting factor in predicting the $q_{T} \rightarrow 0$ behaviour of the distribution at the LHC.

\section{References}

[1] Dokshitzer Yu L, Dyakonov D I and Troyan S I 1980 Phys. Rep. 58269

[2] Parisi G and Petronzio R 1979 Nucl. Phys. B154 427

[3] Collins J, Soper D and Sterman G 1985 Nucl. Phys. B250 199 Collins J and Soper D 1981 Nucl. Phys. B193 381; Erratum 1983 Nucl. Phys. B213 545 Collins J and Soper D 1982 Nucl. Phys. B197 446

[4] Davies C and Stirling W J 1984 Nucl. Phys. B244 337

[5] Ellis R K, Ross D A and Veseli S 1997 Nucl. Phys. B503 309

[6] Ellis R K and Veseli S 1998 Nucl. Phys. B511 649

[7] Frixione S, Nason P and Ridolfi G 1999 Nucl. Phys. B542 311

[8] Kulesza A and Stirling W J 1999 Nucl. Phus. B555 279

[9] Kulesza A and Stirling W J 1999 hep-ph/9909271

[10] Kulesza A and Stirling W J in preparation

[11] D0 collaboration 1999 hep-ex/9909020, submitted to Phys. Rev. Lett. 